\title{
Integrated analysis of differential expression and alternative splicing of non-small cell lung cancer based on RNA sequencing
}

\author{
ZULEI LI $^{1,2}$, KAI ZHAO ${ }^{2}$ and HUI TIAN ${ }^{1}$ \\ ${ }^{1}$ Department of Thoracic Surgery, Qilu Hospital of Shandong University, Jinan, Shandong 250012; \\ ${ }^{2}$ Department of Thoracic Surgery, Zibo Central Hospital, Zibo, Shandong 255036, P.R. China
}

Received February 17, 2016; Accepted March 3, 2017

DOI: $10.3892 / \mathrm{ol} .2017 .6300$

\begin{abstract}
Non-small cell lung cancer (NSCLC) is the most common type of lung cancer, with high morbidity and mortality rates. Numerous diagnosis and treatment methods have been proposed, and the prognosis of NSCLC has improved to a certain extent. However, the mechanisms of NSCLC remain largely unknown, and additional studies are required. In the present study, the RNA sequencing dataset of NSCLC was downloaded from the Gene Expression Omnibus (http://www. ncbi.nlm.nih.gov/geo/). The clean reads obtained from the raw data were mapped to the University of California Santa Cruz human genome (hg19), based on TopHat, and were assembled into transcripts via Cufflink. The differential expression (DE) and differential alternative splicing (DAS) genes were screened out through Cuffdiff and rMATS, respectively. The significantly enriched gene ontology (GO) terms and Kyoto Encyclopedia of Genes and Genomes pathways were obtained through the Database of Annotation, Visualization and Integrated Discovery (DAVID). Different numbers of DE and DAS genes were identified in different types of NSCLC samples, but a number of common functions and pathways were obtained, including biological processes associated with abnormal immune and cell activity. GO terms and pathways associated with substance metabolism, including the insulin signaling pathway and oxidative phosphorylation, were enriched in DAS genes rather than DE genes. Integrated analysis of differential expression and alternative splicing may be helpful in understanding the mechanisms of NSCLC, in addition to its early diagnosis and treatment.
\end{abstract}

\section{Introduction}

Lung cancer is the leading cause of cancer-associated mortality (accounting for $\sim 13 \%$ of all diagnosed cancers), with

Correspondence to: Professor Hui Tian, Department of Thoracic Surgery, Qilu Hospital of Shandong University, 107 Wenhua West Road, Jinan, Shandong 250012, P.R. China

E-mail: huituanqilu@163.com

Key words: non-small cell lung cancer, RNA-sequencing, gene expression omnibus, differential expression, differential alternative splicing
1.8 million new cases in 2012 (1). Between 2011 and 2013, the lung cancer mortality rate increased by $464.84 \%$ in China, and this continued to increase (2). Non-small cell lung cancer (NSCLC) constitutes almost $85 \%$ of all lung cancers and is usually diagnosed an advanced stage (3). Surgery followed by chemoradiotherapy is the most commonly adopted method for locally advanced NSCLC, but the efficacy is diverse across different patients (4). In previous years, immunotherapy became an optional therapeutic for numerous types of cancers, including melanoma and lung cancer, through sustained activation of the immune system $(5,6)$. Certain immune checkpoint inhibitors have been shown to significantly improve the prognosis of a number of cancers, including cytotoxic T-lymphocyte antigen 4 , and nivolumab may prolong the median survival time of patients with melanoma and NSCLC by 2-4 and 6-9 months, respectively (7). However, the morbidity and mortality rate of NSCLC remains high, and additional molecular biology studies are required for more precise treatment and earlier diagnosis.

Next generation RNA sequencing (RNA-Seq) is becoming a popular technology for the quantification of RNA, including mRNA (such as microRNA) and noncoding RNA (such as long noncoding RNA). Variation of genomic structure, including alternative splicing (AS) and single nucleotide polymorphisms, can also be detected by RNA-Seq. Compared with gene microarray, the most evident advantage of RNA-Seq is its ability to detect the expression values of low abundance as well as novel transcripts (8). Previous studies of NSCLC, based on RNA-Seq have been conducted and successfully identified numerous potential targets, involved in the progression or suppression of cancer. Riccardo et al identified the adenosine A3 receptor as a valuable target for NSCLC, based on the combination of gene fusion and differential expression analysis through RNA-Seq (9). In the previous study by Han et al, via RNA-Seq analysis, chromobox 3 and cellular retinoic acid-binding protein 2 were also considered to contribute to the progression of NSCLC (10).

Compared with differential expression analysis at the gene level, exon level analysis may improve analysis of disease stages (11). The present study aimed to identify the potential targets of NSCLC, through the combined analysis of differential expression and AS, and based on the RNA-Seq dataset from the Gene Expression Omnibus (GEO). This may be helpful for the precise treatment of NSCLC, as well as early diagnosis. 


\section{Materials and methods}

RNA-Seq dataset. The RNA-Seq dataset (GSE68795) in the present study was obtained from GEO (http://www.ncbi.nlm. nih.gov/geo/), which was accumulated by Durrans et al (12). A total of 17 samples were included, containing three immature monocytic myeloid cell (IMMC) samples, two epithelial cell (Epi) samples and two neutrophil (Neu) samples from lung cancer patients, as well as three IMMC samples, three Epi samples and four Neu samples from adjacent normal lung tissues. Illumina HiSeq 2000 (Illumina, Inc., San Diego, CA, USA) was used for the sequencing process. Briefly, total RNA was extracted from flow cytometry sorted cells; TruSeq RNA Sample Preparation kit (Illumina, Inc.) was used for the preparation of cDNA libraries from 15-35 ng RNA; cDNA libraries that passed size and purity check were retained for the following sequencing. Single-end 51 bp short sequences (reads) were generated for the IMMC and Neu samples, while paired-end 102 bp reads were generated for the Epi samples, in lung cancer and adjacent normal lung tissues.

Reads mapping and assembling. Quality control of raw reads was conducted using FastQC software version 1.3, which was developed by Andrews (13), with the default parameters, i.e., reads with a quality score $<10$ and $\mathrm{N}>5 \%$ were discarded. The remaining reads were mapped to the UCSC genome (version GRCh37/hg19) through TopHat (2.1.0.Linux_x86_64) (14), a fast splice junction mapper for RNA-Seq reads, with no more than 2 mismatches in 25 bp segments. Cufflinks (14) (2.2.1.Linux_x86_64) was used for the assembly of the mapped reads, which allowed for the identification of novel transcripts, and fragments per kilobase of exon per million fragments mapped (FPKM) representation of gene expression value was obtained.

Differential expression analysis. Cuffdiff of Cufflinks was used to test the significance of differential expression of genes based on FPKM. Genes with fold change (FC) $>2$ (upregulated) or $<0.5$ (downregulated), and false discovery rate (FDR) adjusted for $\mathrm{P}<0.05$, were considered to be differentially expressed.

Differential alternative splicing analysis. The replicate multivariate analysis of transcript splicing (rMATS) (15), developed by Shen et al, was used to screen differential alternative splicing genes across samples. The mapping results (in bam format) were submitted to rMATS. Annotation of genes (in GTF format) was obtained from the UCSC and used for the screening of known splicing sites. Finally, five main alternative splicing types, including skipping exon (SE), retention intron (RI), alternative 5'splice site (A5SS), alternative 3 'splice site (A3SS) and mutually exclusive exons (MXE), which satisfied the criteria of FDR $<0.1$, were screened out as DAS genes.

Functional enrichment analysis. The DE and DAS genes were submitted to the Database for Annotation, Visualization and Integrated Discovery (DAVID; https://david.ncifcrf.gov/) (16) for the analysis of enrichment of gene ontology (GO) terms and Kyoto Encyclopedia of Genes and Genomes (KEGG)

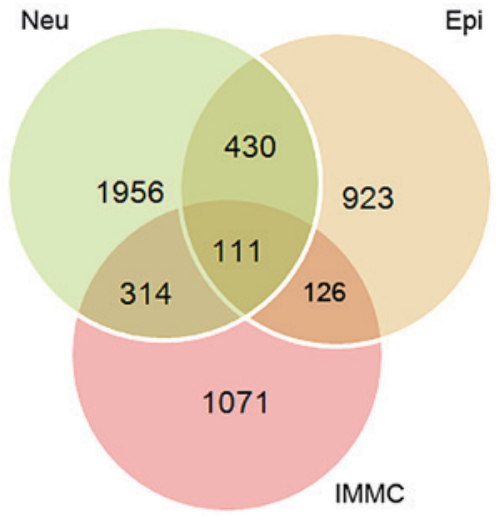

Figure 1. Number of differential expression genes of Epi, IMMC and Neu tumor samples compared with the corresponding normal samples. Epi, epithelial cell; IMMC, immature monocytic myeloid cell; Neu, neutrophil.

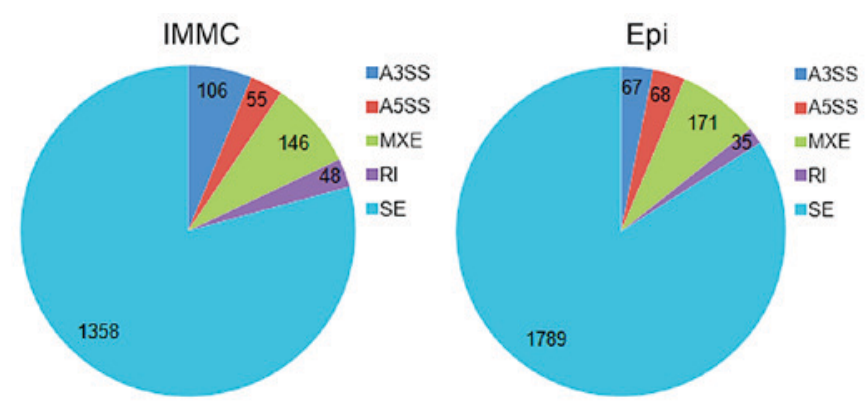

Figure 2. Distribution of different alternative splicing events in Epi and IMMC tumor samples. Epi, epithelial cell; IMMC, immature monocytic myeloid cell; MXE, mutually exclusive exons; RI, retention intron; SE, skipping exon; A3SS, alternative 3'splice site; A5SS, alternative 5'splice site.

pathways. $\mathrm{P}<0.05$ was considered to indicate a statistically significant difference for the screening of significant GO terms and pathways.

\section{Results}

RNA-Seq landscape. The average number of reads obtained from the RNA-Seq dataset was 60242792, and at least 35 million reads were prepared for every sample, as shown in Table I. The clean reads were mapped to the human genome (hg19) by TopHat and $86.50-91.90 \%$ unique mapped rates were obtained across all samples. These were used for the following analysis.

Differential expression analysis. The unique mapped reads were assembled into transcripts by cufflinks, and FPKM values were obtained to quantify the mRNA expression level. Based on the criteria of $\mathrm{FC}>2$ or $<0.5$, and FDR adjusted $\mathrm{P}<0.05$, umerous genes were revealed to be differential expression in tumor samples compared with normal samples.

In Epi tumor samples, a total of 1,624 DE genes were identified, which contained 915 downregulated and 709 upregulated genes. However, in IMMC tumor samples, 1,593 DE genes (639 downregulated and 954 upregulated genes) were obtained. In Neu tumor samples, 1,672 DE genes were obtained, which consisted of 1,141 downregulated and 1,672 upregulated genes. Furthermore, as shown in Fig. 1, 111 overlapping genes were identified among those three groups of DE genes, and 91 of 
Table I. Mapping overview of all the RNA-Sequencing experiments.

\begin{tabular}{lrrrrr}
\hline Sample & All reads & Mapped reads & Mapped rates, $\%$ & Unique mapped & Unique mapped rates, \% \\
\hline Adj-Epi1 & 97717750 & 92115270 & 94.3 & 87061215 & 89.1 \\
Adj-Epi2 & 63907228 & 58725212 & 91.9 & 55272496 & 86.5 \\
Adj-Epi3 & 123019030 & 116594941 & 94.8 & 110088025 & 89.5 \\
Adj-IMM1 & 40304691 & 38809145 & 96.3 & 37030002 & 91.9 \\
Adj-IMM2 & 36685339 & 35109464 & 95.7 & 33284563 & 90.7 \\
Adj-IMM3 & 44679997 & 42067287 & 94.2 & 40025779 & 89.6 \\
Adj-Neu1 & 49967221 & 47183219 & 94.4 & 45452825 & 91.0 \\
Adj-Neu2 & 50458780 & 47159700 & 93.5 & 44872985 & 88.9 \\
Adj-Neu3 & 53402957 & 50511053 & 94.6 & 47590506 & 90.1 \\
Adj-Neu4 & 52277703 & 49603994 & 94.9 & 95969883 & 81.0 \\
Tum-Epi1 & 106711340 & 101588228 & 95.2 & 85487063 & 89.9 \\
Tum-Epi2 & 94931814 & 90391800 & 95.2 & 41037840 & 90.1 \\
Tum-IMM1 & 45344289 & 43038591 & 94.9 & 38638529 & 90.5 \\
Tum-IMM2 & 42060455 & 40620197 & 96.6 & 32229614 & 91.9 \\
Tum-IMM3 & 35570701 & 33745251 & 94.9 & 32442881 & 90.6 \\
Tum-Neu1 & 37106424 & 34076868 & 91.8 & 44980801 & 87.4 \\
Tum-Neu2 & 49981740 & 47119502 & 94.3 & 90.0 \\
\hline
\end{tabular}

Epi, epithelial cell; Adj, adjacent; IMM, immature monocyte; Neu, neutrophil; Tum, tumor.

Table II. Top five most significantly enriched GO terms of differential expression genes of the three groups of non-small cell lung cancer samples.

\begin{tabular}{lll}
\hline Category & \multicolumn{1}{c}{ GO name } & P-value \\
\hline Epi samples & & \\
BP & Response to wounding & $9.39 \times 10^{-15}$ \\
CC & Extracellular space & $8.69 \times 10^{-14}$ \\
BP & Cell adhesion & $2.36 \times 10^{-11}$ \\
BP & Biological adhesion & $2.59 \times 10^{-11}$ \\
CC & Cell fraction & $6.12 \times 10^{-11}$ \\
IMMC samples & & \\
BP & Immune response & $3.22 \times 10^{-10}$ \\
BP & Response to wounding & $3.36 \times 10^{-7}$ \\
BP & Cell division & $2.27 \times 10^{-6}$ \\
CC & Plasma membrane part & $6.03 \times 10^{-6}$ \\
BP & Mitosis & $6.98 \times 10^{-6}$ \\
Neu samples & & \\
BP & Cell adhesion & $1.29 \times 10^{-10}$ \\
BP & Biological adhesion & $1.50 \times 10^{-10}$ \\
MF & GTPase regulator activity & $1.90 \times 10^{-10}$ \\
MF & Nucleoside-triphosphatase & $4.24 \times 10^{-10}$ \\
CC & Plasma membrane part & $5.47 \times 10^{-10}$ \\
\hline
\end{tabular}

$\mathrm{BP}$, biological process; MF, molecular function; CC, cellular component; GO, gene ontology.

those overlaps were consistently upregulated or downregulated across all three types of NSCLC tumor cells.
Table III. Kyoto Encyclopedia of Genes and Genomes pathways of differential expression genes in epithelial cell tumor samples.

\begin{tabular}{lcc}
\hline & $\begin{array}{c}\text { Gene } \\
\text { number }\end{array}$ & P-value \\
\hline ECM-receptor interaction & 29 & $1.64 \times 10^{-9}$ \\
Complement and coagulation cascades & 21 & $4.45 \times 10^{-6}$ \\
Biosynthesis of unsaturated fatty acids & 10 & $1.09 \times 10^{-4}$ \\
Dilated cardiomyopathy & 20 & $1.04 \times 10^{-3}$ \\
Focal adhesion & 34 & $1.51 \times 10^{-3}$ \\
PPAR signaling pathway & 16 & $2.02 \times 10^{-3}$ \\
Hematopoietic cell lineage & 18 & $3.08 \times 10^{-3}$ \\
Pathways in cancer & 48 & $3.29 \times 10^{-3}$ \\
Prion diseases & 10 & $4.92 \times 10^{-3}$ \\
Hypertrophic cardiomyopathy & 17 & $6.63 \times 10^{-3}$ \\
Arrhythmogenic right ventricular & 15 & $1.30 \times 10^{-2}$ \\
cardiomyopathy & & \\
Cell adhesion molecules & 22 & $1.47 \times 10^{-2}$ \\
Ether lipid metabolism & 9 & $1.63 \times 10^{-2}$ \\
Systemic lupus erythematosus & 17 & $2.70 \times 10^{-2}$ \\
Basal cell carcinoma & 11 & $3.58 \times 10^{-2}$ \\
MAPK signaling pathway & 36 & $3.66 \times 10^{-2}$ \\
Synthesis and degradation of & 4 & $4.86 \times 10^{-2}$ \\
ketone bodies & 5 & $4.99 \times 10^{-2}$ \\
Terpenoid backbone biosynthesis & & \\
& & \\
\hline
\end{tabular}

ECM, extracellular matrix; PPAR, peroxisome proliferator-activated receptor; MAPK, mitogen-activated protein kinase. 
Table IV. Kyoto Encyclopedia of Genes and Genomes pathways of differential expression genes in immature monocytic myeloid cell tumor samples.

\begin{tabular}{lcc}
\hline Pathway name & $\begin{array}{c}\text { Gene } \\
\text { number }\end{array}$ & P-value \\
\hline Hematopoietic cell lineage & 25 & $7.50 \times 10^{-7}$ \\
DNA replication & 13 & $7.02 \times 10^{-5}$ \\
Cell cycle & 26 & $2.25 \times 10^{-4}$ \\
Cytokine-cytokine receptor interaction & 42 & $7.78 \times 10^{-4}$ \\
Cell adhesion molecules & 25 & $1.26 \times 10^{-3}$ \\
ECM-receptor interaction & 18 & $1.95 \times 10^{-3}$ \\
Purine metabolism & 25 & $9.05 \times 10^{-3}$ \\
Chemokine signaling pathway & 29 & $9.52 \times 10^{-3}$ \\
Pantothenate and CoA biosynthesis & 6 & $1.02 \times 10^{-2}$ \\
Aminoacyl-tRNA biosynthesis & 10 & $1.29 \times 10^{-2}$ \\
Bladder cancer & 10 & $1.51 \times 10^{-2}$ \\
PPAR signaling pathway & 13 & $2.75 \times 10^{-2}$ \\
Pyrimidine metabolism & 16 & $3.30 \times 10^{-2}$ \\
Viral myocarditis & 13 & $3.36 \times 10^{-2}$ \\
Axon guidance & 20 & $3.44 \times 10^{-2}$ \\
Pathways in cancer & 42 & $3.80 \times 10^{-2}$ \\
Focal adhesion & 28 & $3.92 \times 10^{-2}$ \\
\hline
\end{tabular}

PPAR, peroxisome proliferator-activated receptor; ECM, extracellular matrix; CoA, coenzyme A; tRNA, transfer RNA.

AS in NSCLC tumor samples. Through AS, a mRNA precursor may result in multiple mature mRNAs with different functions. In the present study, the significant DAS genes were identified for the tumor samples compared with the normal ones through rMATS, with the threshold of FDR $<0.1$. Notably, alternative splicing of genes in Neu tumor samples was not found to be significantly different compared with Neu normal samples. A total of 2,130 and 1,713 DAS genes were identified in Epi and IMMC tumor samples, respectively. In total, 272 overlapping genes were screened out between the two lists of DAS genes.

A total of 5 types of AS events were identified, including A3SS, A5SS, MXE, RI and SE. As shown in Fig. 2, SE is the most prevalent AS event in Epi and IMMC tumor samples, and accounts for 84.00 and $79.28 \%$ of all the AS events in Epi and IMMC, respectively. RI is the least prevalent AS event, which only accounts for 1.64 and $2.80 \%$ of all the AS events in Epi and IMMC, respectively.

GO and pathway analysis. Functional enrichment analysis based on the DAVID identified a number of significantly enriched GO terms and KEGG pathways in DE and DAS genes. The top five most significant GO terms of DE genes of the three groups of NSCLC tumor samples are listed in Table II. As shown in Table II, biological processes associated with the cell and immune system were significantly enriched $(\mathrm{P}<0.05)$ in the DE genes of all the three types of NSCLC tumor samples. Enriched pathways of DE genes of Epi, IMMC and Neu tumor samples are listed in Tables III, IV and V, respectively. Similar to the enriched GO terms, those pathways were mainly involved
Table V. Kyoto Encyclopedia of Genes and Genomes pathways of differential expression genes in neutrophil tumor samples.

\begin{tabular}{|c|c|c|}
\hline Pathway name & $\begin{array}{l}\text { Gene } \\
\text { number }\end{array}$ & P-value \\
\hline Focal adhesion & 61 & $4.81 \times 10^{-7}$ \\
\hline Regulation of actin cytoskeleton & 62 & $2.59 \times 10^{-6}$ \\
\hline Fc gamma R-mediated phagocytosis & 34 & $5.94 \times 10^{-6}$ \\
\hline Chemokine signaling pathway & 53 & $2.67 \times 10^{-5}$ \\
\hline Leukocyte transendothelial migration & 37 & $5.92 \times 10^{-5}$ \\
\hline ECM-receptor interaction & 29 & $7.03 \times 10^{-5}$ \\
\hline Axon guidance & 37 & $4.28 \times 10^{-4}$ \\
\hline Pathogenic Escherichia coli infection & 20 & $1.01 \times 10^{-3}$ \\
\hline Hypertrophic cardiomyopathy & 26 & $1.40 \times 10^{-3}$ \\
\hline Adherens junction & 24 & $1.71 \times 10^{-3}$ \\
\hline Pathways in cancer & 73 & $2.62 \times 10^{-3}$ \\
\hline Dilated cardiomyopathy & 26 & $4.57 \times 10^{-3}$ \\
\hline $\begin{array}{l}\text { Valine, leucine and isoleucine } \\
\text { degradation }\end{array}$ & 15 & $7.22 \times 10^{-3}$ \\
\hline Glioma & 19 & $8.70 \times 10^{-3}$ \\
\hline $\begin{array}{l}\text { Natural killer cell mediated } \\
\text { cytotoxicity }\end{array}$ & 32 & 0.018518 \\
\hline Non-small cell lung cancer & 16 & $2.06 \times 10^{-2}$ \\
\hline Gap junction & 23 & $2.30 \times 10^{-2}$ \\
\hline $\begin{array}{l}\text { Aldosterone-regulated sodium } \\
\text { reabsorption }\end{array}$ & 13 & $2.45 \times 10^{-2}$ \\
\hline Endocytosis & 41 & $2.55 \times 10^{-2}$ \\
\hline MAPK signaling pathway & 56 & $2.78 \times 10^{-2}$ \\
\hline $\begin{array}{l}\text { Progesterone-mediated oocyte } \\
\text { maturation }\end{array}$ & 22 & $2.95 \times 10^{-2}$ \\
\hline $\begin{array}{l}\text { Arrhythmogenic right ventricular } \\
\text { cardiomyopathy }\end{array}$ & 20 & $2.97 \times 10^{-2}$ \\
\hline ErbB signaling pathway & 22 & $3.32 \times 10^{-2}$ \\
\hline Fc epsilon RI signaling pathway & 20 & $3.81 \times 10^{-2}$ \\
\hline p53 signaling pathway & 18 & $3.84 \times 10^{-2}$ \\
\hline Colorectal cancer & 21 & $4.23 \times 10^{-2}$ \\
\hline Cell adhesion molecules & 30 & $4.58 \times 10^{-2}$ \\
\hline Glycerolipid metabolism & 13 & $4.84 \times 10^{-2}$ \\
\hline B cell receptor signaling pathway & 19 & $4.89 \times 10^{-2}$ \\
\hline
\end{tabular}

ECM, extracellular matrix; MAPK, mitogen-activated protein kinase.

in the dysregulation of cell division and abnormal activity of the immune and inflammatory systems.

For DAS genes in Epi and IMMC tumor samples, their enriched GO terms are shown in Fig. 3, which was obtained by submitting the DAVID result to the WEGO website (http://wego.genomics.org.cn/cgi-bin/wego/index. pl), and their enriched KEGG pathways are shown in Fig. 4. As expected, those DAS genes were also involved in cell and immune abnormality. The insulin signaling pathway and oxidative phosphorylation, which were associated with substance metabolism and were not revealed by DE genes, were also found to be significantly enriched $(\mathrm{P}<0.05)$ in Epi and IMMC tumor samples, respectively. DE and DAS genes 
A

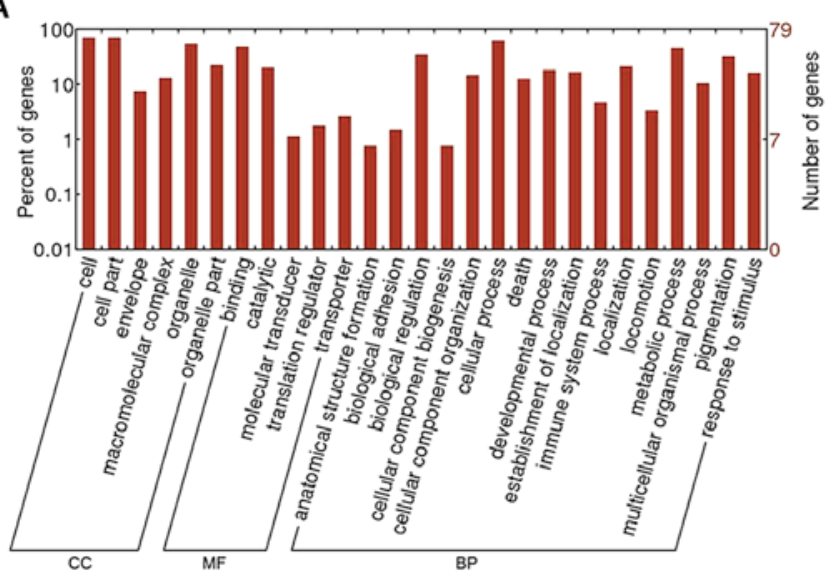

B

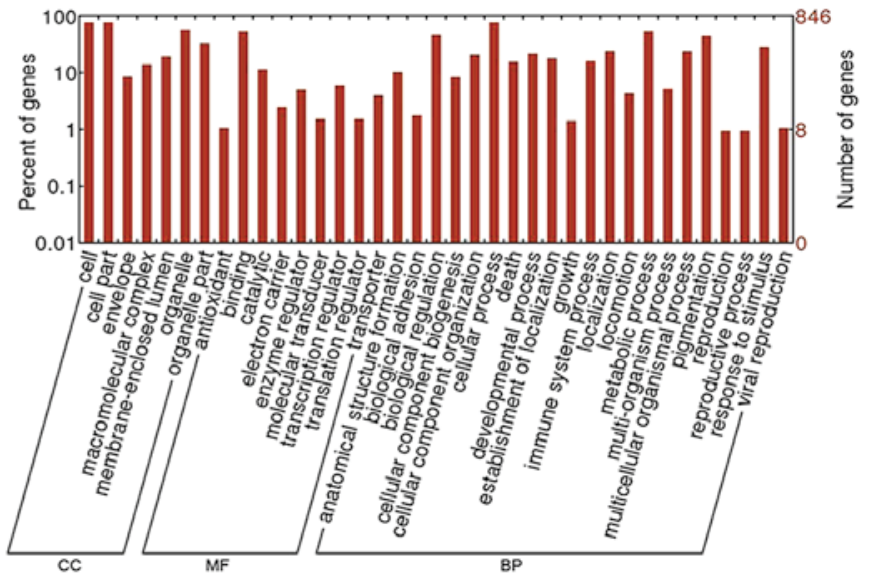

Figure 3. Significantly enriched gene ontology terms of alternative splicing genes in (A) epithelial cell and (B) immature monocytic myeloid cell tumor samples. BP, biological process; MF, molecular function; $\mathrm{CC}$, cellular component.

A

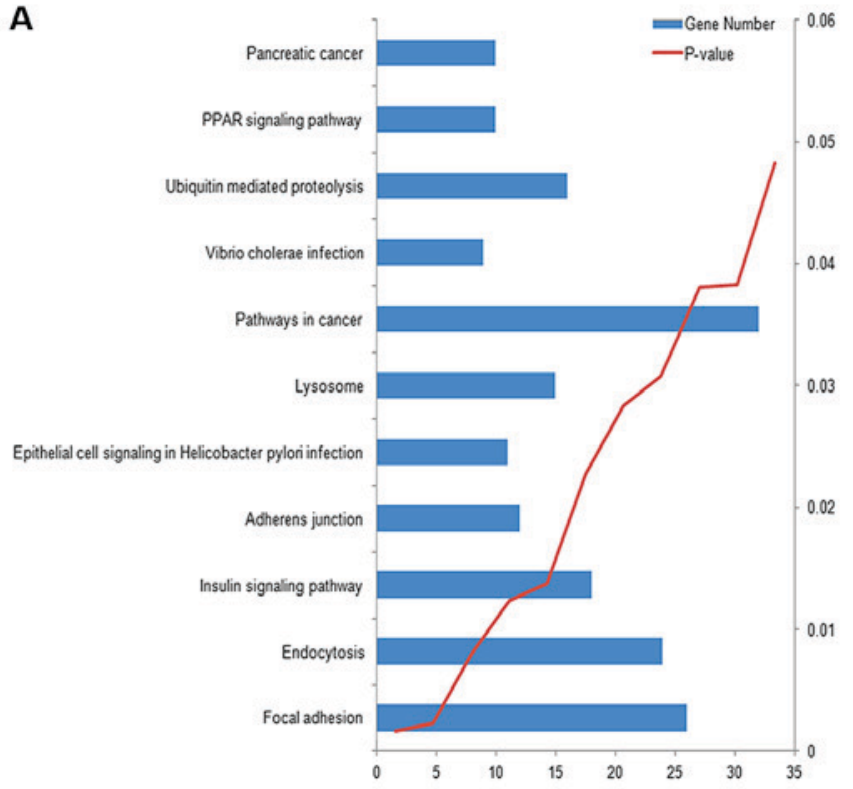

B

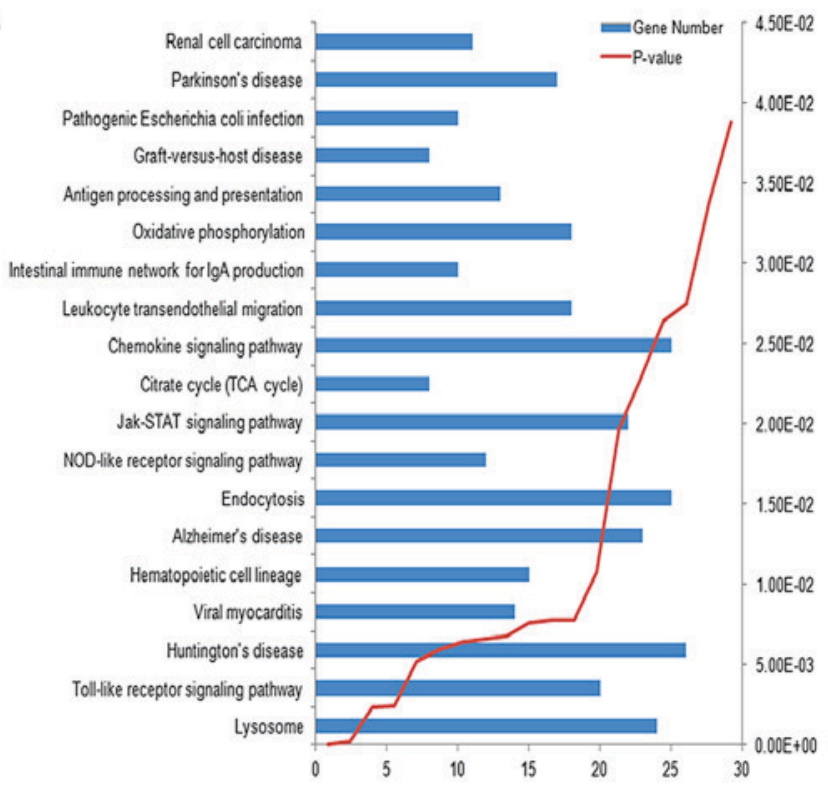

Figure 4. Significantly enriched Kyoto Encyclopedia of Genes and Genomes pathways of alternative splicing genes in (A) epithelial cell and (B) immature monocytic myeloid cell tumor samples. PPAR, peroxisome proliferator-activated receptor; IgA, immunoglobulin A; Jak-STAT, Janus kinase-signal transducers and activators of transcription; NOD, non-obese diabetic; TCA, tricarboxylic acid.

may be complementary in the understanding of mechanisms of NSCLC.

\section{Discussion}

Lung cancer remains the leading cause of cancer-associated mortality in recent years, and its main type is NSCLC (17). An increasing number of studies about NSCLC have been emerging and numerous potential biomarkers for the early diagnosis or treatment of NSCLC have been identified subsequent to the development of molecular biology technologies. However, the mechanisms of NSCLC remain largely unknown, and additional studies are required to improve its prognosis. In the present study, through re-analysis of the RNA-Seq dataset from the GEO database, the significant DE and DAS genes were screened out in three types of NSCLC tumor samples (Epi, IMMC and
Neu), compared with the corresponding normal samples, and their enriched GO terms and KEGG pathways were obtained. The present study aimed to provide results to help with the development of therapeutic methods and drugs for NSCLC.

The rapid growth of RNA-Seq provides the chance to observe variation in the genome at the exon level, and screening of alternative splicing is one of its important applications (18). AS is an important mechanism that could result in proteins with different functions from one mRNA precursor, and it has been shown to affect the status of numerous diseases. AS events involving exons 15-19 of d'Origine nantais tyrosine kinase have been screened out in lung cancer (19). In addition, the T cell factor $4 \mathrm{~K}$ AS isoform was found to improve the prognosis of NSCLC by suppressing the proliferation and metastasis of tumor cells (20). In the present study, significant alternative splicing was not observed in any genes in the Neu 
tumor samples compared with the normal samples. Numerous AS genes were identified in Epi and IMMC tumor samples, which may indicate the differences of those three types of cells in the inducement of NSCLC.

In accordance with previous studies, SE is the most prevalent AS event, while RI is the least prevalent AS event in Epi and IMMC tumor samples $(21,22)$. A total of 272 genes demonstrated alternative splicing in Epi and IMMC tumor samples, and this may be an indication of the important roles of those genes in the progression of NSCLC. Compared with AS analysis, numerous DE genes were identified in all three types of tumor samples, and 111 overlapping genes were screened out. One of the significant differences between the DE genes and DAS genes is the involvement of substance metabolism-associated pathways of DAS genes, including the insulin signaling pathway and oxidative phosphorylation. Insulin-like growth factor (IGF-1R) performs important roles through its downstream signaling in a number of solid tumors, including NSCLC (23). Furthermore, IGF-1R affects cisplatin and radiation resistance in lung cancer (24). In addition, oxidative stress/activity is affected by IGF-1R in numerous diseases, resulting in the dysregulation of biosystems, such as in cancer or neurodegeneration $(25,26)$. DE and DAS analysis revealed a number of common GO terms and pathways, and the primary terms and pathways were cell or immune-associated. These have been investigated in numerous studies and were confirmed to play important roles in the development of numerous types of cancers, including lung cancer, gastric cancer and liver cancer (27-29).

Based on the integrated analysis of DE and DAS, alternative splicing was identified by leucine-rich repeat kinase 2 (LRRK2) in Epi and IMMC tumor samples, and was consistently downregulated in all three types of tumor cells. LRRK2 is a member of the leucine-rich repeat kinase family and encodes a protein with an ankryin repeat region. LRRK2 has been shown to play important roles in neurodegeneration diseases, including Parkinson's disease (30-32). The role of LRRK2 in cancer development remains unclear; however, certain indirect associations were identified (33). For example, through disrupting the activation of the proto-oncogene MET, LRRK2 may prevent tumor cell proliferation (34). Therefore, it may be a novel target for NSCLC.

In conclusion, through the combined analysis of DE and DAS, potential targets for NSCLC were screened out. Furthermore, DE and DAS may be complementary for understanding the mechanisms of NSCLC. The present study may be helpful in the diagnosis and treatment of NSCLC.

\section{References}

1. He X, WangJ and Li Y: Efficacy and safety of docetaxel for advanced non-small-cell lung cancer: A meta-analysis of Phase III randomized controlled trials. Onco Targets Ther 8: 2023-2031, 2015.

2. She J, Yang P, Hong Q and Bai C: Lung cancer in China: Challenges and interventions. Chest 143: 1117-1126, 2013.

3. Santarpia M, González-Cao M, ViteriS,Karachaliou N, Altavilla G and Rosell R: Programmed cell death protein-1/programmed cel death ligand-1 pathway inhibition and predictive biomarkers: Understanding transforming growth factor-beta role. Transl Lung Cancer Res 4: 728-742, 2015.
4. Nakajima Y, Akiyama H, Kinoshita H, Atari M, Fukuhara M, Saito Y, Sakai $\mathrm{H}$ and Uramoto H: Case report of two patients having successful surgery for lung cancer after treatment for Grade 2 radiation pneumonitis. Ann Med Surg (Lond) 5: 1-4, 2015

5. Wieder T, Brenner E, Braumüller $\mathrm{H}$ and Röcken $\mathrm{M}$ : Immunotherapy of melanoma: Efficacy and mode of action. J Dtsch Dermatol Ges 14: 28-37, 2016

6. Pilotto S, Molina-Vila MA, Karachaliou N, Carbognin L, Viteri S, González-Cao M, Bria E, Tortora G and Rosell R: Integrating the molecular background of targeted therapy and immunotherapy in lung cancer: A way to explore the impact of mutational landscape on tumor immunogenicity. Transl Lung Cancer Res 4: 721-727, 2015.

7. Kobold S, Duewell P, Schnurr M, Subklewe M, Rothenfusser S and Endres S: Immunotherapy in tumors. Dtsch Arztebl Int 112: 809-815, 2015.

8. Morozova O and Marra MA: Applications of next-generation sequencing technologies in functional genomics. Genomics 92: 255-264, 2008.

9. Riccardo F, Arigoni M, Buson G, Zago E, Iezzi M, Longo D, Carrara M, Fiore A, Nuzzo S, Bicciato S, et al: Characterization of a genetic mouse model of lung cancer: A promise to identify non-small cell lung cancer therapeutic targets and biomarkers. BMC Genomics 15 (Suppl 3): 1, 2014.

10. Han SS, Kim WJ, Hong Y, Hong SH, Lee SJ, Ryu DR, Lee W, Cho YH, Lee S, Ryu YJ, et al: RNA sequencing identifies novel markers of non-small cell lung cancer. Lung Cancer 84: 229-235, 2014.

11. Bisognin A, Pizzini S, Perilli L, Esposito G, Mocellin S, Nitti D, Zanovello P, Bortoluzzi S and Mandruzzato S: An integrative framework identifies alternative splicing events in colorectal cancer development. Mol Oncol 8: 129-141, 2014.

12. Durrans A, Gao D, Gupta R, Fischer KR, Choi H, El Rayes T, Ryu S, Nasar A, Spinelli CF, Andrews W, et al: Identification of reprogrammed myeloid cell transcriptomes in NSCLC. PLoS One 10: e0129123, 2015.

13. Andrews S: FastQC: A quality control tool for high throughput sequence data. http://www.bioinformatics.babraham.ac.uk/projects/fastqc/. Accessed January 10, 2016.

14. Trapnell C, Roberts A, Goff L, Pertea G, Kim D, Kelley DR, Pimentel H, Salzberg SL, Rinn JL and Pachter L: Differential gene and transcript expression analysis of RNA-seq experiments with TopHat and Cufflinks. Nat Protoc 7: 562-578, 2012

15. Shen S, Park JW, Lu ZX, Lin L, Henry MD, Wu YN, Zhou Q and Xing Y: rMATS: Robust and flexible detection of differential alternative splicing from replicate RNA-Seq data. Proc Natl Acad Sci USA 111: E5593-E5601, 2014.

16. Huang da W, Sherman BT and Lempicki RA: Systematic and integrative analysis of large gene lists using DAVID bioinformatics resources. Nat Protoc 4: 44-57, 2009.

17. Sang Y, Bi X, Liu Y, Zhang W and Wang D: Adverse prognostic impact of TGFB1 T869C polymorphism in non-small-cell lung cancer. Onco Targets Ther 10: 1513-1518, 2017.

18. Han Y, Gao S, Muegge K, Zhang W and Zhou B: Advanced applications of RNA sequencing and challenges. Bioinform Biol Insights 9 (Suppl 1): 29-46, 2015

19. Krishnaswamy S, Mohammed AK, Amer OE, Tripathi G, Alokail MS and Al-Daghri NM: Novel splicing variants of recepteur d'origine nantais (RON) tyrosine kinase involving exons 15-19 in lung cancer. Lung Cancer 92: 41-46, 2016.

20. Fan YC, Min L, Chen H and Liu YL: Alternative splicing isoform of T cell factor $4 \mathrm{~K}$ suppresses the proliferation and metastasis of non-small cell lung cancer cells. Genet Mol Res 14: 14009-14018, 2015.

21. Wang X, Xu X, Lu X, Zhang Y and Pan W: Transcriptome Bioinformatical analysis of vertebrate stages of Schistosoma japonicum reveals alternative splicing events. PLoS One 10: e0138470, 2015.

22. Hide WA, Babenko VN, van Heusden PA, Seoighe C and Kelso JF: The contribution of exon-skipping events on chromosome 22 to protein coding diversity. Genome Res 11: 1848-1853, 2001.

23. Scagliotti GV and Novello S: The role of the insulin-like growth factor signaling pathway in non-small cell lung cancer and other solid tumors. Cancer Treat Rev 38: 292-302, 2012.

24. Sun Y, Zheng S, Torossian A, Speirs CK, Schleicher S, Giacalone NJ, Carbone DP, Zhao Z and Lu B: Role of insulin-like growth factor-1 signaling pathway in cisplatin-resistant lung cancer cells. Int J Radiat Oncol Biol Phys 82: e563-e572, 2012.

25. Dávila D, Fernández S and Torres-Alemán I: Astrocyte resilience to oxidative stress induced by insulin-like growth factor I (IGF-I) involves preserved AKT (protein kinase B) activity. J Biol Chem 291: 2510-2523, 2016. 
26. Khawaja T, Chokshi A, Ji R, Kato TS, Xu K, Zizola C, Wu C, Forman DE, Ota T, Kennel P, et al: Ventricular assist device implantation improves skeletal muscle function, oxidative capacity, and growth hormone/insulin-like growth factor-1 axis signaling in patients with advanced heart failure. J Cachexia Sarcopenia Muscle 5: 297-305, 2014.

27. De Palma M: The role of the immune system in cancer: From mechanisms to clinical applications. Biochim Biophys Acta 1865: $1-2,2016$

28. Soto-Ortiz L and Finley SD: A cancer treatment based on synergy between anti-angiogenic and immune cell therapies. J Theor Biol 394: 197-211, 2016.

29. Boros LG, D'Agostino DP, Katz HE, Roth JP, Meuillet EJ and Somlyai G: Submolecular regulation of cell transformation by deuterium depleting water exchange reactions in the tricarboxylic acid substrate cycle. Med Hypotheses 87: 69-74, 2016.

30. Oosterveld LP, Allen JC Jr, Ng EY, Seah SH, Tay KY, Au WL, Tan EK and Tan LC: Greater motor progression in patients with Parkinson disease who carry LRRK2 risk variants. Neurology 85: 1039-1042, 2015.
31. Marder K, Wang Y, Alcalay RN, Mejia-Santana H, Tang MX, Lee A, Raymond D, Mirelman A, Saunders-Pullman R, Clark L, et al: Age-specific penetrance of LRRK2 G2019S in the Michael J. Fox Ashkenazi Jewish LRRK2 consortium. Neurology 85: 89-95, 2015.

32. Lee JW and Cannon JR: LRRK2 mutations and neurotoxicant susceptibility. Exp Biol Med (Maywood) 240: 752-759, 2015.

33. Bae JR and Lee BD: Function and dysfunction of leucine-rich repeat kinase 2 (LRRK2): Parkinson's disease and beyond. BMB Reports 48: 243-248, 2015.

34. Looyenga BD, Furge KA, Dykema KJ, Koeman J, Swiatek PJ, Giordano TJ, West AB, Resau JH, Teh BT and MacKeigan JP: Chromosomal amplification of leucine-rich repeat kinase-2 (LRRK2) is required for oncogenic MET signaling in papillary renal and thyroid carcinomas. Proc Natl Acad Sci USA 108: 1439-1444, 2011. 